\title{
A case of COVID-19 with the atypical CT finding
}

\author{
Ryota Kurimoto $^{1}$, Kenji Sakurai ${ }^{1}$, Ken Motoori ${ }^{1}$, and Akira Suda ${ }^{1}$ \\ ${ }^{1}$ Tsudanuma Central General Hospital
}

April 27, 2020

\begin{abstract}
We report a case of COVID-19 with only atypical CT findings; tree-in-bud appearance and centri-lobular nodules. Some COVID-19 cases show only atypical CT findings. Physicians should make comprehensive judgements.
\end{abstract}

\section{Key Clinical Message}

We report a case of COVID-19 with only atypical CT findings; tree-in-bud appearance and centri-lobular nodules. Some COVID-19 cases show only atypical CT findings. Physicians should make comprehensive judgements.

\section{Main Text}

A 30-year-old man presented with a 2-day-history of fatigue, fever (39.0) and cough without sputum and respiratory failure. 6 days ago, he had participated the banquet and meeting in Tokyo, in which COVID-19 had been spreading. Laboratory tests showed elevated C-reactive protein $(4.23 \mathrm{mg} / \mathrm{dL})$ without the specific findings about any pathogen. Chest CT revealed only tree-in-bud appearance on the right upper-lobe (Fig.1B) and centri-lobular nodules with unclear edge on the left lower lobe without typical ground-glass opacity (GGO) as COVID-19 (Fig. 1A-C). Diagnosis of COVID-19 was made by RT-PCR from nasopharyngeal swab. He has received supportive care and the symptoms have improved.

COVID-19 usually demonstrates the specific pattern of Chest CT-findings, such as GGO, inverted-halo sign, and so on $[1,2]$. However, it had been reported that several cases showed only atypical findings or no significant findings in Chest CT [1,2]. Although the role of CT images for COVID-19 is extremely high, it is also important to recognize that some cases show only atypical findings in CT, especially at a diagnosis. We should not decide whether or not to carry out PCR test based on CT findings alone. At the time, we physicians should make comprehensive judgement for this pandemic situation.

\section{Author contribution}

RK, KS, KM and AS contributed to conception, design and data collection. RK wrote the paper. All authors discussed about this report.

\section{References}

1. Adam Bernheim, Xueyan Mei, Mingqian Huang, et al. Chest CT Findings in Coronavirus Disease-19 (COVID-19): Relationship to Duration of Infection. Radiology. 2020 Feb 20:200463

2. Scott Simpson, Fernando U. Kay, Suhny Abbara, et al. Radiological Society of North America Expert Consensus Statement on Reporting Chest CT Findings Related to COVID-19. Endorsed by the Society of Thoracic Radiology, the American College of Radiology, and RSNA. Radiology: Cardiothoracic Imaging $20202: 2$. 


\section{Figure legend}

\section{Figure. Representative images of Chest CT}

A. Chest $\mathrm{X}$ ray image.

B, C. Chest CT image. Tree-in-bud appearance were seen in the right upper lobe (B) and centri-lobular nodules with unclear edge were seen in the left lower lobe.

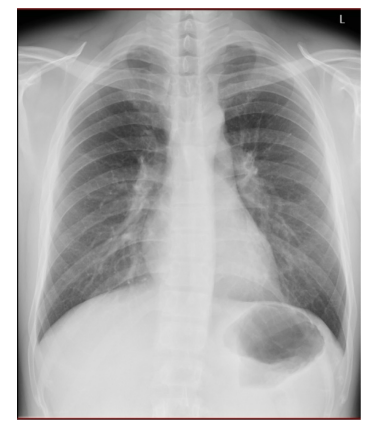

$\frac{A}{B} \mid C$
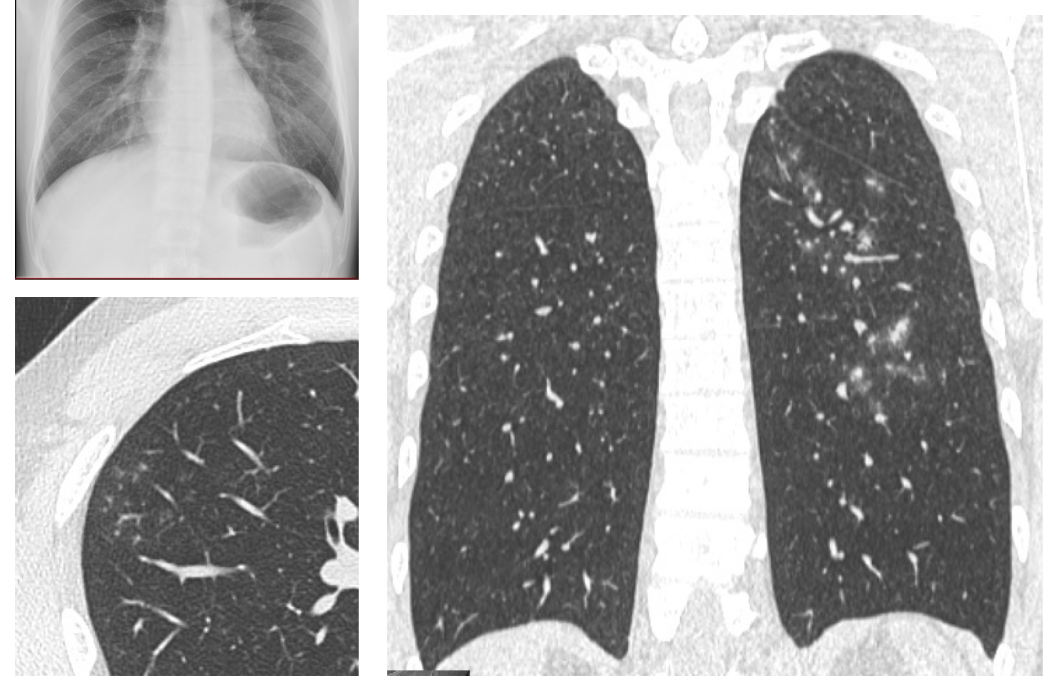